\title{
Non-linearity correction of PMT response on the observed particle densities in GRAPES-3
}

\author{
A. Chandra* ${ }^{* a, b}$, S. Ahmad ${ }^{a, c}$, M. Chakraborty ${ }^{a, b}$, S.R. Dugad ${ }^{a, b}$, S.K. Gupta ${ }^{a, b}$, B. \\ HariHaran $^{a, b}$, Y. Hayashi ${ }^{a, g}$, S.S.R. Inbanathan ${ }^{a, d}$, P. Jagadeesan ${ }^{a, b}$, A. Jain ${ }^{a, b}$, P. \\ Jain $^{a, e}$, V.B. Jhansi ${ }^{a, b}$, S. Kawakami ${ }^{a, g}$, H. Kojima ${ }^{a, h}$, S. Mahapatra ${ }^{a, f}$, P.K. Mohanty ${ }^{a, b}$, \\ S.D. Morris ${ }^{a, b}$, P.K. Nayak ${ }^{a, b}$, A. Oshima ${ }^{a, i}$, D. Pattanaik ${ }^{a, b}$, P.S. Rakshe ${ }^{a, b}$, K. \\ Ramesh $^{a, b}$, B.S. Rao ${ }^{a, b}$, L.V. Reddy ${ }^{a, b}$, S. Shibata ${ }^{a, i}$, F. Varsi ${ }^{a, e}$, M. Zuberi ${ }^{a, b}$ \\ ${ }^{a}$ GRAPES-3 Experiment, Cosmic Ray Laboratory, Raj Bhavan, Ooty 643 001, India. \\ ${ }^{b}$ Tata Institute of Fundamental Research, Homi Bhabha Road, Mumbai 400 005, India. \\ ${ }^{c}$ Department of Physics, Aligarh Muslim University, Aligarh 202 002, India. \\ ${ }^{d}$ The American College, Madurai 625 002, India. \\ ${ }^{e}$ Indian Institute of Technology, Kanpur, 208016 India. \\ ${ }^{f}$ Department of Physics, Utkal University, Bhubneswar 751 004, India. \\ ${ }^{g}$ Graduate School of Science, Osaka City University, Osaka 558-8585, Japan \\ ${ }^{h}$ Faculty of Engineering, Aichi Insitute of Technology, Toyota City, Aichi 470-0392, Japan \\ ${ }^{i}$ College of Engineering, Chubu University, Kasugai, Aichi 487-8501, Japan \\ E-mail: anuj.hep@gmail.com
}

\begin{abstract}
The GRAPES-3 (Gamma Ray Astronomy at PeV EnergieS-3) experiment has been designed to study the composition and energy spectrum of primary cosmic rays (PCRs) over TeV - PeV range. It is equipped with 400 plastic scintillators covering an area of $25000 \mathrm{~m}^{2}$ and a muon detector spread in an area of $560 \mathrm{~m}^{2}$. The precise measurement of particle densities from each detector is very important for any air shower experiment. Photo-multiplier tubes (PMT) are widely used as a transducer to collect the light produced in detector medium to electric signals. These signals are then processed to measure the particle densities. However, these PMTs tend to show nonlinearity and saturation at higher signals due to the large shower core landing near the detector. The GRAPES-3 detectors record $\sim(50-70)$ particles in the linear range. This results in improper shower size estimation near the knee energy. In order to overcome this situation and to estimate the correct shower size, a systematic approach has been devised and demonstrated to correct the particle density beyond the linear range. It is seen from the method allows us to estimate particle densities $\sim(500-800)$ which can improve the measurements beyond knee.
\end{abstract}

36th International Cosmic Ray Conference -ICRC2019-

July 24th - August 1st, 2019

Madison, WI, U.S.A.

\footnotetext{
* Speaker.
} 


\section{Introduction}

High energy cosmic rays (CRs) are believed to be the messenger from the outer galaxies. The study of cosmic rays may reveal the signatures about the occurrence of various astronomical phenomena such as pulsars, supernovae explosions, active galactic nuclei and etc. Cosmic rays encounter complex magnetic field in inter-galactic and extra-galactic space and get accelerated. The understanding of the nature of their source and acceleration to the ultra-high energies (UHE) $E \geq 10^{15} \mathrm{eV}(\mathrm{PeV})$ are still eluding us instead of some progress shown by theoretical and experimental fronts [1]. The phenomena involved up to PeV energies can be explained with the help of shock acceleration of Fermi mechanism coupled with supernovae explosions. The higher energies CRs are modeled with the help of extended version of same model with the concepts of shock progressing through stellar winds and acceleration through multiple shocks within the galactic disk. However, the power law energy spectrum of all-particle exhibits change in the spectral index from -2.7 to -3.0 at $\sim 3 \mathrm{PeV}$ and opens-up the debate for important checks for the models explain the propagation and acceleration of CRs. The acceleration mechanism accompanies the change in the composition of CRs at around the knee energy and favors the scenario of supernova shock into stellar wind of a progenitor star for higher energies [2]. Therefore, the composition studies are sought to understand the phenomena occurring around the knee energies.

There are various investigations performed by direct $[3,4,5,6,7,8,9]$ and indirect observations on primary cosmic rays around the $\mathrm{PeV}$ energies. The continuous falling flux of primary CRs limits the detection of high energies CRs by the space based experiments. On the other hand, the detection of extensive air shower (EAS) by ground based experiments require larger area and longer exposure time to understand the very high energy $\left(>10^{15} \mathrm{eV}\right) \mathrm{CRs}$. Some of the large area ground based experiments CASA [10, 11], Tibet AS $\gamma$ [12], KASCADE [13, 14], MILAGRO [15] and ARGO-YBJ [16] have shown their measurements up to and above knee energies.

The electrons, in an air shower is the most studied component for getting information on the shower, particularly for generating trigger, determining arrival direction and estimating the energy of the primary particle. A measurement of the lateral (radial) density distribution of particles in a shower using an array of unshielded charged particle detectors provides a good estimate of the observed number of particles in the shower,

$$
N_{e}^{o b s}=2 \pi r \rho_{e}(r) d r
$$

where $\rho_{e}(r)$ is the density of particles at a distance $\mathrm{r}$ from the core of the shower. With the core of the shower incident within the shower array, $r_{1}$ is generally taken to be 0 . The distance $r_{2}$ depends on the size of the shower array but the observed lateral distribution function is generally extrapolated to $r_{2}=\infty$ to obtain the total number $N_{e}^{t o t}$ of particles in the shower. Despite considerable fluctuations in the development of individual showers in the atmosphere and the differences in development profile for different primary nuclei, the shower size $\left(N_{e}^{o b s}\right.$ or $\left.N_{e}^{\text {tot }}\right)$ is known to be a good parameter for estimating primary energy of showers.

Therefore, it is required to estimate the particle density precisely is required by any air shower experiments. The secondary particles produced in EAS is an increasing function of PCRs energy [17]. In order to study the $\mathrm{CRs}$ of PeV energies demands the estimation of secondary particles to be observed very precisely. Gamma Ray Astronomy at PeV Energies Phase-3 (GRAPES-3) array 
of plastic scintillator detectors equipped with photo multiplier tubes (PMTs). While primaries of $\sim$ $\mathrm{PeV}$ energies generates a large number of secondary particles in a shower and therefore, PMTs gets saturated followed by the non-linear behavior of PMT in case of such large showers. The extension of the particle densities is already attempted [18] by using 2-PMT detectors. Particle densities observed by $\sim 75 \%$ of the detectors are still limited due to the constraint of PMTs non-linearity and saturation and therefore, an attempt is made to correct the non linearity observed up-to certain extent.

\section{GRAPES-3 Experiment}

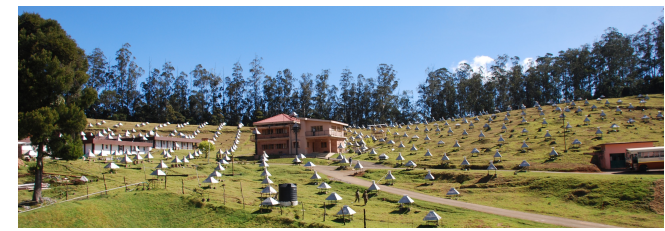

(a) Array Layout

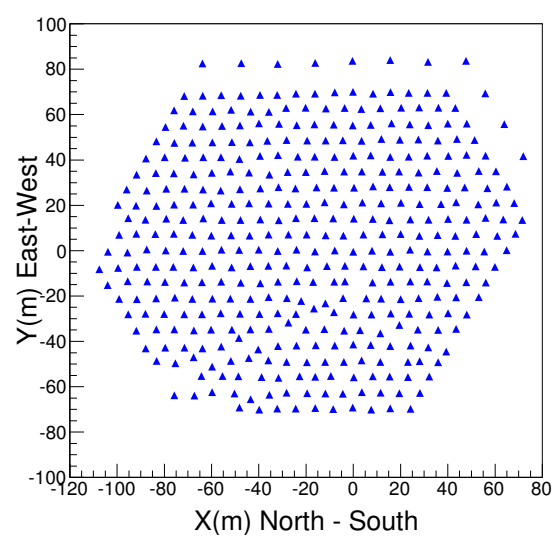

(b) Array Map

Figure 1: Plastic scintillation detectors deployed in hexagons rings over $25,000 \mathrm{~m}^{2}$ area.



(a) 4 Muon Stations

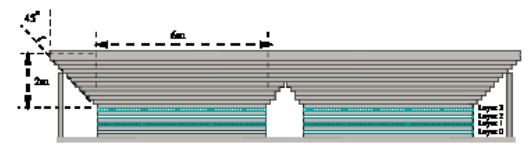

(b) Cross sectional view of module inside one station

Figure 2: Muon Detectors

The GRAPES-3 (Gamma Ray Astronomy at PeV Energies Phase-3) experiment is assembled with a densely packed EAS array at Ooty in India $\left(11.4^{\circ} \mathrm{N}, 76.7^{\circ} \mathrm{E}, 2200 \mathrm{~m}\right)$. Currently, it comprises $\sim 400$ plastic scintillation detectors[2] each of $1 \mathrm{~m}^{2}$ area and the layout is shown in Fig.1. These shower detectors are laid in hexagonal structured array as a building block of a unit of 3 scintillator detectors making an equilateral triangle with $8 \mathrm{~m}$ inter detector separation. This combination of $8 \mathrm{~m}$ separation with an altitude $\sim 2.2 \mathrm{~km}$ at Ooty $\left(\sim 800 \mathrm{~g} . \mathrm{cm}^{-2}\right)$ can push the threshold of shower triggering energy as low as $\sim 30 \mathrm{TeV}$ [19], which can work as a bridge between direct and indirect measurements. In addition, the muon detector consists of 16 modules with each module $35 \mathrm{~m}^{2}$ in area and built with 4-orthogonal layers and each layer is of 58 proportional counters. Each proportional counter is $6 \mathrm{~m}$ long with $10 \times 10 \mathrm{~cm}^{2}$ cross-sectional area. Alternate layers of 
counters are arranged orthogonal and are separated by $15 \mathrm{~cm}$ thick concrete layer build of blocks. A thick layer of absorber (concrete $\sim 550 \mathrm{~g} . \mathrm{cm}^{-2}$ ) above the $1^{\text {st }}$ counter layer provides the filter for muons of energy $\geq 1 \mathrm{GeV}$. A cross-section of two adjacent muon detector modules is shown in FIGURE: $2 \mathrm{a}$ and $2 \mathrm{~b}$.

\subsection{Types of Shower Detectors}

Shower detectors which have been developed in house at Ooty and are followed by various R\&D activities can be categorized as follows:

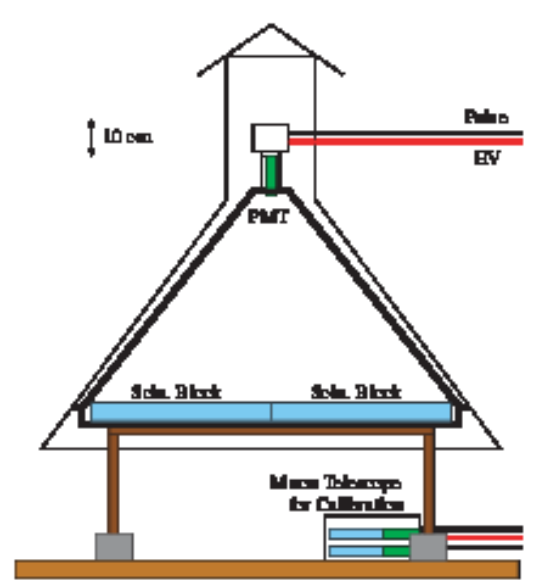

Figure 3: Schemetic of a shower detector, including the padels used for calibration

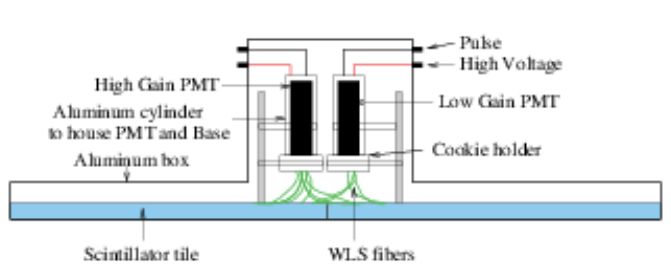

Figure 4: Cross sectional view of 2-PMT Fiber Detector

- Cone Detectors: The cone type of scintillation detectors are the earliest configuration, being used in the GRAPES-3 experiment [2]. All of them are of $1 \mathrm{~m}^{2}$ each and having four 50 $\times 50 \mathrm{~cm}^{2}$ area and $5 \mathrm{~cm}$ thick plastic scintallator blocks placed on the base of a trapezoidal shaped aluminum cone. A photo multiplier tube (PMT) ETL-9907SB02 of $5 \mathrm{~cm}$ diameter glass window is placed at $60 \mathrm{~cm}$ above the surface of scintillator blocks heading it's window towards scintillator blocks as shown in FIGURE: 3 and other details are given therein[2].

- Fiber Detectors: Due to the indirect detection of diffused photons from the wall of container in cone type detectors there are significant loss of signal, whereas most of the cone type scintillator detectors have a photon output of $\leq 10$ photo-electrons[20]. On the other hand, the geometry of the detector also resulted in a large spatial non-uniformity in the signal across the detector and a variation $\sim 30 \%$ is observed in photon output from the center to the edge of the detector. In order to overcome these limitations. we have developed the modified configuration of scintillation detectors by using wave-length shifting (WLS) fibers and the advantages of using WLS are mentioned in the work of P.K. Mohanty et al.[20]. The saturation of the PMTs encountered at very high particle densities in the earlier GRAPES-3 detectors, limited their use over a wider range of primary cosmic ray energies. To overcome the limitation of saturation of PMT, the WLS fiber detectors have been designed with two PMTs as shown in FigurE: 4. The main design goal for the new detector is to efficiently 
record shower particles over a large dynamic range ( 1 - 5000 particles), to cover a wide range in the energy spectrum of the primary cosmic rays from $10^{13} \mathrm{eV}$, where the particle density at the core is rather small; to $10^{17} \mathrm{eV}$ where densities may reach several thousand particles $\mathrm{m}^{-2}$. Efficient detection of a single or a few particles is ensured by designing a detector with high photon yield and good spatial uniformity. The high photon yield also implies that the PMT may be operated at a relatively lower voltage, thereby reducing the noise and increasing its life. The PMTs are typically operated at a gain of (3-5) $\times 10^{6}$, which is within the recommended region specified by the manufacturer. However, at high particle densities, the anode current become very large and the PMT response become non-linear. For the ETL-9807B PMT used here, the response becomes non-linear at a peak anode current of $50-150 \mathrm{~mA}$. This translates into the onset of the non-linearity at $\gtrsim 50$ particles and of saturation at $\sim 100-200$ particles. This introduces error in the estimation of the various shower parameters. Although, one can use the signal from an earlier stage of the dynode chain to avoid saturation, we prefer to employ a second PMT to enhance the dynamic range of our detectors. The configuration of 2-PMT detectors is explained in [20]. Presently, there are 105 double PMT WLS fiber detectors have been deployed in the array.

\section{Observed Particle Density Spectrum}

The particle densities generated in large air showers observed by HG-PMTs are limited due to it's non-linear and saturation effects. The response of a PMT starts to become non-linear when a large number of photons are detected. The anode current becomes very high [20] due to enhancement in the production of secondary electrons through the dynodes operated at succesive higher potential [21]. The presence of large number of electrons reduces the strength of electric field between the two dynodes. Therefore, the output signal of the PMT will be smaller as compared to

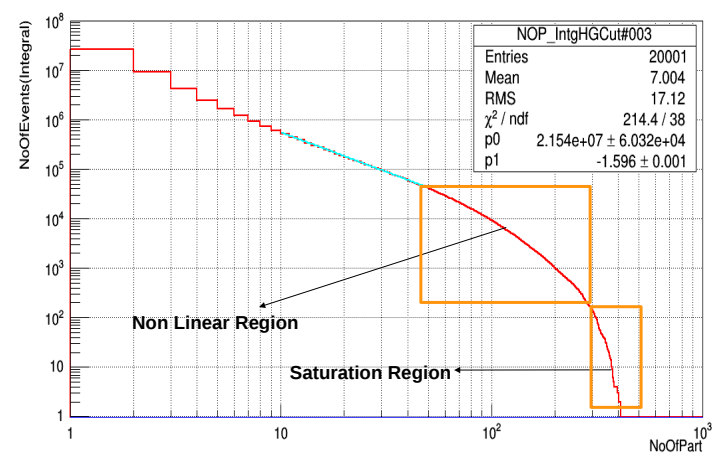

Figure 5: Non-Linearity and Saturation of high-gain PMT

the expected signal [22]. As the number of photons incident on the PMT keep increasing, the PMT starts to saturate and at one point, the output signal amplitude becomes independent of the input number of photons. A detailed explanation on non-linearity and saturation behavior of PMTs can be found in the manuals provided by the manufacturers [23, 24]. As shown in FIGURE: 5, all the high-gain PMTs used in the GRAPES-3 experiment exhibit onset of non-linearity at $\sim(50-100)$ particles and saturation in the range of $\sim(100-300)$ particles. 


\section{Methodology}

To develop a correction method, it is required to know the spectrum expected in the nonlinear region. Since the low-gain PMT measures the same particles as the high-gain PMT being in the same detector, the density spectrum obtained by low-gain PMT can be used for the expected spectrum at higher particle densities. Spectral slope of density spectrum for 105 low-gain PMT detectors were obtained by fitting a power-law function in the range of 50 to 5000 particles with full one year data. A mean value of -1.58 for spectral index within the statistical uncertainty is obtained from all 105 dual-PMT detectors.

A power law function given in the following equation was used for extrapolation of density spectrum in the non-linear region of the high-gain PMT beyond 50 particle

$$
Y=A_{50} \times\left(\frac{x}{50}\right)^{-\gamma}
$$

Where, $A_{50}$ is the number of events $\gtrsim 50$ particle, $\gamma$ is the spectral slope obtained from extended particle density spectrum observed by low-gain PMTs which is $1.58, \mathrm{x}$ is the number of particles $>50$. Next, the corresponding integral number of events was computed using equation 4.1. This extrapolated spectrum along with the observed high-gain PMT particle density spectrum is shown in Figure:6.

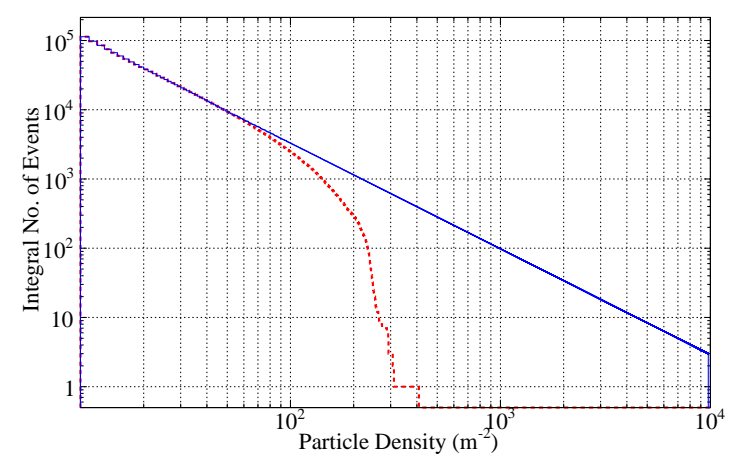

Figure 6: High-gain and Extrapolated particle density spectrum.

In this methodology, the observed integral number of events at a given particle density to be corrected is compared with the number of integral events from the extrapolated particle density spectrum. Thereafter, the particle $m^{-2}$ is transferred to the particle density at the compared integral events of extended density spectrum. More clearly, the corrected density can be evaluated by using the function explained as in equation 4.1 where, $\mathrm{A}$ is again the integral number of events at 50 particle $\mathrm{m}^{-2}$, and $\mathrm{x}$ in the integral number of events at particle density to be corrected from non linear region of high-gain PMT. The limit upto particle density to be corrected is decided as 300 particles $\mathrm{m}^{-2}$ for the zeroth order approximation. Therefore, the density is being corrected in the range of $50<$ particle density $\lesssim 300$. The corrected particle density as a function of uncorrected particle density is shown in FIGURE: 7. As expected the dependence is not linear and beyond uncorrected particle density of $\gtrsim 200-250$, the variation is rapid. The correction for the large particles $>(200-250)$ may not be accurate since the particle density to be corrected are lying in 
the saturation region of high-gain PMT. Therefore, a limit has been taken in order to fix a boundary between the non-linear and saturation region of high-gain PMT. The limit is decided by the value of ratio of corrected particle density to the value of uncorrected particle density. The region with ratio value $\lesssim 2.76$ is being called as the region of non-linearity and higher than 2.76 is called the saturation region.

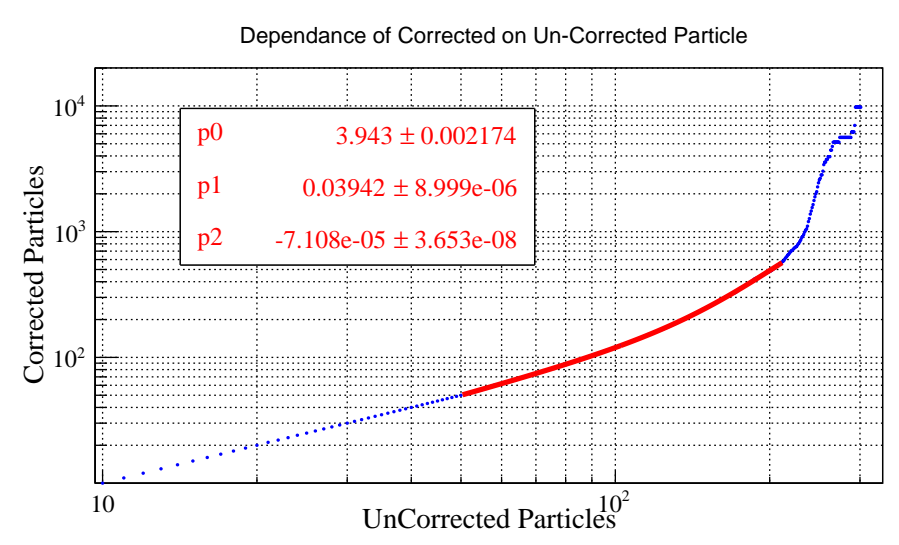

Figure 7: Dependance of corrected particle density on uncorrected particle density.

The variation of the corrected particle density versus uncorrected particle density is shown in FIGURE: 7, The errors are also introduced to each corrected particle density as a statistical error considered from the number of integral events from the distribution of uncorrected particle density. This dependence of corrected particle density on uncorrected particle density is modeled with a slowly varying exponential function having three free parameters as given in the following equation.

$$
Y=50+A \times\left(e^{\left(B(x-50)+C(x-50)^{2}\right)}-1\right)
$$

Where $\mathrm{A}, \mathrm{B}$ and $\mathrm{C}$ are the free parameters, $\mathrm{x}$ in the uncorrected particle density and $\mathrm{y}$ is the value of corrected particle density estimated empirically. The values of free parameters A, B and C are evaluated after fitting the dependence of corrected particle density on uncorrected particle density in the region of non-linearity of the high-gain PMT.

\section{Corrected Particle Density Spectrum}

The correction of particle density observed in non-linear region of a PMT requires the estimation of free parameters A, B and C. Thereafter, only the correction for non-linear particle density can be applied on event-by-event basis for the recorded air shower events to all the high-gain PMTs. The previous section described the method for evaluating the parameters A, B, and C. The analysis was carried out for 2017. The parameters that obtained for a month was used for the correction for the events for the same month. The particles density observed in non-linear region are corrected on event-by-event basis. In FIGURE: 8, the non-linearity observed and corrected density spectrum are shown for one of the shower detector. The corrected spectrum clearly shows a significant extension of observed particle density spectrum. 


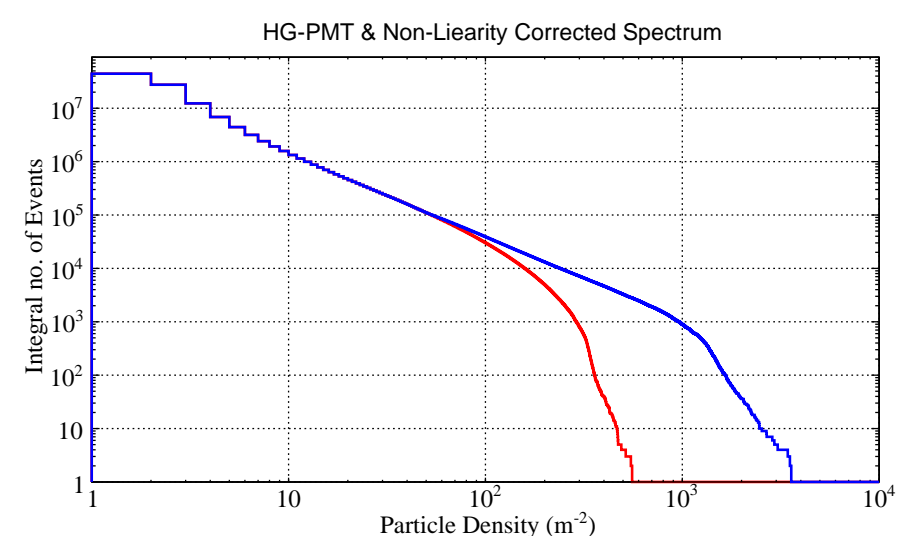

Figure 8: Corrected and observed particle densities in non-linear range.

\section{Conclusions}

The corrected integral particle density spectrum shows a significant correction and the correction method has improved the particle densities $\sim(500-800)$ particles. Earlier we were bound to use particle density observed in the linear range $\sim(50-70)$ particles.

\section{References}

[1] V.S. Berezinskiï, et al., Astrophysics of Cosmic Rays, North-Holland, Amsterdam, 1990.

[2] S.K. Gupta et al., Nuclear Instruments and Methods in Physics Research A 540 (2005) 311323.

[3] K. Asakimori, et al., Astrophys. J. 502 (1998) 278.

[4] M. Boezio et al., Astropart. Phys. 19 (2003) 583.

[5] Haino et al., Phys. Lett. B 594 (2004) 35.

[6] Y.S. Yoon et al., The Astrophys. J. 728 (2011) 122.

[7] M. Aguilar et al., Phys. Rev. Lett. 114, 171103 (2015).

[8] O. Adriani et al., Science 332 (2011) 69.

[9] J. Alcaraz et al., Phys. Lett. B 494 (2000) 193.

[10] D.J. Bird et al., 24 ${ }^{\text {th }}$ ICRC-1995, Vol. 2, August 28-September 8, 1995 in Rome, Italy. Edited by N. Iucci and E. Lamanna. International Union of Pure and Applied Physics, 1995., p.760.

[11] L. Fortson, Proceedings of the 26th ICRC-1999, August 17-25, 1999. Salt Lake City, Utah, USA. Under the auspices of the International Union of Pure and Applied Physics (IUPAP). Volume 3. Edited by D. Kieda, M. Salamon, and B. Dingus, p.125.

[12] M. Amenomori, et al., Nucl. Instr. and Meth. A 288 (1990) 619.

[13] T. Antoni, et al., Nucl. Instr. and Meth. A 513 (2003) 490;

T. Antoni, et al., Astropart. Phys. 14 (2001) 245.

[14] A. Haungs et al, Journal of Physics: Conference Series 409 (2013) 012005 
[15] R. Atkins, et al., Nucl. Instr. and Meth. A 449 (2000) 478;

R. Atkins, et al., Astrophys. J. 595 (2003) 803.

[16] C. Bassi, et al., Nucl. Instr. and Meth. A 443 (2000) 342;

C. Bassi, et al., Astropart. Phys. 17 (2002) 151.

[17] A.A. Radu, et al., Romanian Reports in Physics, Vol. 60, No. 1, P. 45 - 55, 2008.

[18] A. Chandra et al., PoS (ICRC2017) 479.

[19] S.C. Tonwar, et al., Nuclear Physics B (Proc. Suppl.) 151 (2006) 477 - 480.

[20] P.K. Mohanty et al., Astroparticle Physics 31 (2009) 24 - 36.

[21] https://www.hamamatsu.com/resources/pdf/etd/PMT_handbook_v3aE-Chapter4.pdf

[22] X. Wang et al., Chinese Physics C 40, 8 (2016) 086003.

[23] PhototMultiplier Tubes Basics and Applications, $3^{r d}$ Edition, 2007, $160-161$.

[24] http ://et-enterprises.com/images/datasheets/9807B.pdf 